\title{
Variable response to radioactive iodine treatment in poorly differentiated thyroid carcinoma
}

\author{
Hunter J. Underwood ${ }^{1}$, Ashok R. Shaha ${ }^{2}$, Kepal N. Patel ${ }^{1}$ \\ ${ }^{1}$ Division of Endocrine Surgery, NYU Langone Health, New York, NY, USA; ${ }^{2}$ Head and Neck Service, Memorial Sloan-Kettering Cancer Center, \\ New York, NY, USA \\ Correspondence to: Kepal N. Patel, MD. Division of Endocrine Surgery, NYU Langone Health, $5301^{\text {st }}$ Ave, Suite 6H, New York, NY 10016, USA. \\ Email: Kepal.Patel@nyulangone.org. \\ Provenance: This is an invited article commissioned by the Section Editor De-tao Yin (Professor and Supervisor of Postgraduate, Department of \\ Thyroid Surgery, the First Affiliated Hospital of Zhengzhou University, Zhengzhou, China). \\ Comment on: Nikitski AV, Rominski SL, Condello V, et al. Mouse Model of Thyroid Cancer Progression and Dedifferentiation Driven by STRN- \\ ALK Expression and Loss of p53: Evidence for the Existence of Two Types of Poorly Differentiated Carcinoma. Thyroid 2019;29:1425-37.
}

Submitted Oct 16, 2019. Accepted for publication Oct 21, 2019.

doi: 10.21037 /gs.2019.10.14

View this article at: http://dx.doi.org/10.21037/gs.2019.10.14

The global incidence of thyroid cancer has steadily increased, however mortality has remained unchanged as most thyroid malignancies are well-differentiated (WDTC) with indolent behavior. The incidence, however, of poorly differentiated (PDTC) and anaplastic thyroid cancer (ATC) have also increased proportionally and these are aggressive tumors associated with significant morbidity and mortality (1). PDTC has been recognized as a distinct entity that represents an intermediate position in the sequence of de-differentiation from WDTC to ATC with unique histopathology, molecular abnormalities, and diagnostic criteria (2).

Thyroid carcinoma is a continuum of diseases, where at one end there is papillary thyroid cancer, the human cancer with one of the best prognoses, a long term survival $>98 \%$. On the other end of the spectrum is ATC, the deadliest human cancer, with almost $99 \%$ mortality. It is an amazing and biological enigma that in the same human organ we see this huge variation. The molecular biology of this disease may help us to understand this spectrum and help in providing targets for therapy.

The development of PDTC is hypothesized to be secondary to a "multi-hit" process that includes both early and late driver mutations (3). Early drivers most commonly include mutations in $B R A F$ or $R A S$ and have been well described in the molecular pathogenesis of WDTC. Later drivers, which are also seen in ATC, include TP53 and TERT. Mouse models have been created previously in attempts to study the dedifferentiation process based on an early point mutation (BRAF V600E) with later TP53 inactivation, however they rapidly progressed and often skipped PDTC stage (4).

For this reason, Nikitski et al. recently developed a novel mouse model utilizing $S T R N-A L K$ gene fusion as the early driver mutation, which has not been previously described (5). Transgenic mice (STRN-ALK; p53KO) were created to investigate the effect of STRN-ALK expression combined with loss of p53 in the de-differentiation pathway. The mice were divided by age group (6-, 12-, and 18-month) and maintained on a normal diet or on goitrogen treatment. Thyroid tumors were initially detected using ultrasonography. At 12 months, $48 \%$ of the mice on a regular diet and $86 \%$ of mice treated with goitrogen developed confirmed thyroid cancers. Irrespective of goitrogen treatment, thyroid cancers in the mice showed aggressive histologic features and lung metastasis was found in $8(24 \%)$ of tumor-bearing mice.

Thyroid tumors in the mice included WDTC $(n=26)$, PDTC $(n=21)$, and ATC $(n=8)$. All PDTC tumors met Turin diagnostic criteria, however further histological analysis of the PDTC tumors lead to the identification of two subtypes with unique histologic and immunohistochemical characteristics. PDTC1 tumors had a lower N:C ratio and preserved stronger immunoreactivity for thyroglobulin and E-cadherin. PDTC2 tumors had a higher N:C 
ratio and pronounced loss of thyroid differentiation on immunohistochemistry. The gene expression profiles of the different subtypes were investigated using RNA-Seq and qRT-PCR for further validation. The principal component analysis of tumor transcriptional profiles showed the PDTC1 and PDTC2 clustered separately from each other, as well as from benign thyroid and WDTC. The expression levels of genes involved in thyroid differentiation, iodine uptake, and metabolism (e.g., $T g, T p o, A n o 1$, and Duox2) were significantly decreased in PDTC2, but not PDTC1. Notably, the expression of Slc5as (NIS) was significantly lower in both types of PDTC when compared to normal thyroid tissue, however the average expression was approximately two-fold lower in PDTC2.

This work demonstrates a novel mouse model that clearly demonstrates the dedifferentiation of thyroid cancer and introduces the possibility of two unique PDTC subtypes for the first time. The distinction of PDTC subtypes at the molecular level, particularly in regards to expression of Slc5as (NIS), is of great interest given the potential clinical implications for radioactive iodine (RAI) avidity in these high-risk tumors within humans. These findings provide genetic evidence to explain the variability of response to RAI treatment that has been clinically observed in human PDTC $(6,7)$. Further work is needed to evaluate the functionality of NIS expression in PDTC1 and PDTC2 by specifically examining RAI uptake within these tumors, however this study represents huge strides in our understanding of the molecular genetics of lethal thyroid tumors.

\section{Acknowledgments}

None.

\section{Footnote}

Conflicts of Interest: The authors have no conflicts of interest to declare.

Ethical Statement: The authors are accountable for all aspects of the work in ensuring that questions related to the accuracy or integrity of any part of the work are appropriately investigated and resolved.

\section{References}

1. Janz TA, Neskey DM, Nguyen SA, et al. Is the incidence of anaplastic thyroid cancer increasing: A population based epidemiology study. World J Otorhinolaryngol Head Neck Surg 2018;5:34-40.

2. Nikiforov YE. Genetic alterations involved in the transition from well-differentiated to poorly differentiated and anaplastic thyroid carcinomas. Endocr Pathol 2004;15:319-27.

3. Sadow PM, Faquin WC. Poorly differentiated thyroid carcinoma: an incubating entity. Front Endocrinol (Lausanne) 2012;3:77.

4. McFadden DG, Vernon A, Santiago PM, et al. p53 constrains progression to anaplastic thyroid carcinoma in a Braf-mutant mouse model of papillary thyroid cancer. Proc Natl Acad Sci U S A 2014;111:E1600-9.

5. Nikitski AV, Rominski SL, Condello V, et al. Mouse Model of Thyroid Cancer Progression and Dedifferentiation Driven by STRN-ALK Expression and Loss of p53: Evidence for the Existence of Two Types of Poorly Differentiated Carcinoma. Thyroid 2019;29:1425-37.

6. Patel KN, Shaha AR. Poorly differentiated and anaplastic thyroid cancer. Cancer Control 2006;13:119-28.

7. Lin JD, Chao TC, Hsueh C. Clinical characteristics of poorly differentiated thyroid carcinomas compared with those of classical papillary thyroid carcinomas. Clin Endocrinol (Oxf) 2007;66:224-8.
Cite this article as: Underwood HJ, Shaha AR, Patel KN. Variable response to radioactive iodine treatment in poorly differentiated thyroid carcinoma. Gland Surg 2019;8(6):589590. doi: 10.21037/gs.2019.10.14 